\title{
Oser, unir, réussir
}

\section{Christoph Bosshard}

Dr, vice-président de la FMH, responsable du département Données, démographie et qualité (DDQ)

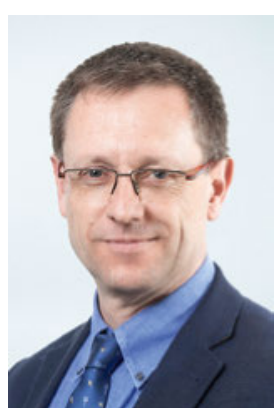

Tout le monde parle de directives, de prise en charge coordonnée et d'interprofessionnalité. La FMH ne fait pas qu'en parler, elle s'y attelle avec le projet interprofessionnel de l'ASQM sur l'itinéraire clinique des patients atteints du cancer colorectal. Pour ce projet clairement ambitieux, l'ASQM est arrivée à réunir 20 organisations spécialisées, pour moitié des organisations non médicales, et à trouver un consensus. Voici une brève rétrospective du chemin parcouru.

Après avoir étudié la littérature scientifique internationale en préparation à ce projet et alors que nous étions en train de consulter différents experts de la question, nous nous sommes demandé si nous n'étions pas fous. Un itinéraire clinique qui allie l'interprofessionnalité aux différents secteurs de soins n'était pas seulement considéré comme un projet pionnier au niveau international mais aussi comme un challenge. Et pourtant nous nous y sommes risqué, à l'image du proverbe africain si tu veux aller vite, marche seul mais si tu veux aller loin, marchons ensemble. Et cela en valait la peine: nous avons gagné un prix international dans le cadre de l'European Clinical Pathway Congress mais surtout construit une communauté performante avec les organisations impliquées.

\section{Si tu veux aller vite, marche seul mais si tu veux aller loin, marchons ensemble.}

Les enquêtes préliminaires nous ont permis d'avoir une première vue d'ensemble des schémas de traitements et d'investigations existant dans différentes régions ou institutions en rapport avec notre projet. L'itinéraire clinique interprofessionnel des patients atteints du cancer colorectal a vu le jour après des travaux intenses de coordination et de recherche de consensus. Vous trouverez un compte rendu détaillé de ce projet dans le présent numéro.

L'heure est venue de saluer cette étape importante mais aussi d'adresser nos plus vifs remerciements aux personnes et organisations qui ont rendu cette aventure possible. Pour leur courage et leur persévérance d'avoir suivi ce chemin avec nous. Pour leur précieux travail et les connaissances partagées. Pour la motiva- tion et l'initiative, toujours aussi vives, de poursuivre sur cette voie. Ce n'était pas une évidence au vu des efforts et des dépenses consentis jusqu'à présent: toutes les heures non rémunérées des membres de l'équipe de projet aussi bien des organisations de professionnels de santé que de la FMH s'élèvent à près de CHF 700000. Là aussi, nous leur adressons toute notre reconnaissance.

La prochaine étape consiste à analyser l'impact d'un tel itinéraire de patients. Le dépistage précoce, les investigations et le traitement seront-ils améliorés? Son introduction entraînera-t-elle un renchérissement, les coûts

\section{Les interventions clé de l'itinéraire}

clinique doivent être prises en considération au moment de fixer les fichiers cantonaux.

resteront-ils équivalents ou économiserons-nous des ressources grâce à une utilisation coordonnée et efficace des moyens utilisés? Jusqu'ici, nous avions émis l'hypothèse qu'un meilleur suivi des guidelines et des itinéraires cliniques augmentait l'efficience de notre système de santé. Il s'agit maintenant de le vérifier. Ce projet va nous réclamer au-delà de nos seules ressources. A tous les niveaux, nous aurons là aussi besoin de courage pour permettre des changements. La direction du projet va bientôt concrétiser les prochaines étapes. Je continue de compter sur la force de notre communauté interprofessionnelle mais j'attends aussi un soutien du côté des assureurs, de l'administration et des milieux politiques. Les conditions cadres qu'il s'agit de mettre en place englobent une tarification conforme à la loi des prestations définies dans l'itinéraire des patients mais aussi la prise en considération des interventions clé du même itinéraire clinique, au moment de fixer les fichiers cantonaux pour les registres des maladies oncologiques.

Nous avons matière à nous réjouir aujourd'hui mais de nombreux défis nous attendent aussi demain. Ensemble, nous y arriverons. Je remercie tous ceux - et en particulier les 20 organisations concernées et les membres de l'équipe de projet - qui nous ont soutenus et qui continueront de nous soutenir. 